CASE REPORT

\title{
Compartment syndrome after thrombolysis for acute myocardial infarction
}

\author{
A Reuben, E Clouting
}

Emerg Med J 2005;22:77. doi: 10.1136/emj.2003.004960

The use of various forms of thrombolytic treatment in acute myocardial infarction has been widespread for many years and their potential to cause haemorrhagic complications well recognised. Haemorrhagic sequaelae are usually minor and consist of oozing from venepuncture or cannula sites or minor haemorrhage from mucosal membranes. The potential for more serious bleeding complications is acknowledged, but is fortunately less common. Isolated cases of compartment syndrome have been reported in the past, but these usually follow some degree of local trauma. The case is reported of a patient who developed atraumatic compartment syndrome of the thigh after single dose thrombolysis.

A previously healthy but sedentary 61 year old man presented to the emergency department in November 2001 after an episode of chest pain. He described a several hour history of crushing central pain and an electrocardiograph (ECG) performed shortly after arrival showed ST segment elevation in the inferior leads and accordingly the diagnosis of inferior myocardial infarction was made. Treatment was carried out according to a standard protocol and having already received $300 \mathrm{mg}$ aspirin, he was given streptokinase 1.5 million units intravenously less than half an hour after arrival. Within two hours he began to complain of some discomfort in his left leg, describing a dull ache localised to the anterior aspect of his thigh with no obvious precipitants and no history of trauma or intramuscular injection. Examination at this point was unremarkable, with no swelling or focal tenderness and no evidence of distal neurovascular compromise. He was treated with simple analgesia and the pain settled. A further two hours later the patient was reviewed when the pain returned and this time failed to settle. Examination now showed an obviously swollen upper leg, with a tense anterior compartment but soft posteriorly with normal pedal pulses and sensation. This raised the concern of possible spontaneous haemorrhage in to the anterior fascial compartment of his thigh with increasing intra-compartmental pressures. He was reviewed by the on call orthopaedic team who felt there was a high index of suspicion of a developing compartment syndrome. He was taken to theatre within the hour where he underwent a fasciotomy of the anterior fascial compartment of his left thigh under a regional nerve block. The operative findings were of a tense anterior compartment full of fresh blood that was incised and released.

Postoperatively he made a good recovery and his symptoms settled rapidly. His wound was closed at 48 hours and he was given prophylactic intravenous antibiotics. His further stay was uneventful and he was discharged home one week later.

\section{DISCUSSION}

The beneficial effects of thrombolysis in acute myocardial infarction have been recognised for many years. ${ }^{1}$ Early treatment with streptokinase in selected cases has been shown to have significant long term benefits on morbidity and mortality. ${ }^{2}$ Streptokinase is an enzyme made by haemolytic streptococci, which activates plasminogen by an interaction with its endogenous pro-activator, resulting in fibrinolysis. Bleeding is therefore a well recognised complication of thrombolysis, but is usually minor with up to $70 \%$ having oozing at venepuncture sites. ${ }^{3}$ Non-cerebral bleeds have been found in $4.5 \%$ of patients after streptokinase and intracerebral bleeds in $0.4 \%{ }^{4}$ Predictors of increased risk of bleeding after thrombolysis are increasing age, low body weight, female sex, choice of thrombolytic agent, and African ancestry. ${ }^{5}$ Management of bleeding is generally supportive. Serious bleeding calls for withdrawal of the thrombolytic and may require administration of coagulation factors and antifibrinolytic drugs (aprotinin or tranexamic acid). Compartment syndrome occurs when there is an increase in pressure within a sealed fascial compartment after some form of insult. As the pressure rises a cascade of events is set up, culminating in increasing pressure, reduced venous flow, and tissue ischaemia. Clinical diagnosis is often difficult. While there is clearly potential for spontaneous bleeding within a fascial compartment after thrombolytic therapy, spontaneous haemorrhage after treatment with a single thrombolytic agent has not previously been reported. This case highlights the need to maintain a high index of suspicion of spontaneous bleeding from any site after thrombolysis.

\section{Authors' affiliations}

A Reuben, E Clouting, Emergency Department, Bristol Royal Hospital for Children, Bristol, UK

Correspondence to: $\operatorname{Dr} A$ Reuben, Emergency Department, Bristol Royal Hospital for Children, Paul O'Gorman Building, Bristol, UK; adamreuben@hotmail.com

Accepted for publication 22 May 2003

\section{REFERENCES}

1 Chesebro JH, Knatterud G, Roberts R, et al. Thrombolysis in myocardial infarction (TIMI) trial, phase 1: comparison between intravenous tissue plasminogen activator and intravenous Sstreptokinase. Circulation 1987:76:142-54.

2 Fibrinolytic Therapy Trialists' (FTT) Collaborative Group. Indications for fibrinolytic therapy in suspected acute myocardial infarction: collaborative overview of early mortality and major morbidity results from all randomised trials of more than 1000 patients. Lancet 1994;343:311-22.

3 Yusuf S, Cairns AJ. Evidence based cardiology. London: BMJ Books, 1998.

4 ISIS-3 Collaborative Group. A randomised comparison of streptokinase Vs TPA Vs anistreplase and of aspirin plus heparin $V_{s}$ aspirin alone among 41,299 cases of suspected acute myocardial infarction. Lancet 1992;339:753.

5 Berkowitz SD, Granger CB, Pieper KS. Incidence and predictors of bleeding after contemporary thrombolytic therapy for myocardial infarction. Circulation 1997;95:2508-16. 